\title{
Calculation of Foster-type Equivalent Network for Stripline 45 Degrees Bend Based on Novel Calculation Method of the Eigenmode
}

\author{
Takaharu HIRAOKA Kazuhiro HAMATANI Jui-Pang HSU
}

Faculty of Engineering, Kanagawa University, Yokohama Japan

\begin{abstract}
Foster-type equivalent network for stripline 45 degrees bend of equal or different stripline width is derived based on eigenmode method. The key step is how to calculate eigenmodes of the planar junction of $45^{\circ}$ bend up to necessary mode number with exactness. We propose a new idea of three stage cascade-connected stripline configuration and double symmetry with magnetic wall, which is practically carried out with success. The derived equivalent network is applied to the calculation of frequency characteristics and field distribution under the operating.

Index Terms - Stripline 45 degrees bend, 3-stage stripline circuit, double symmetry with magnetic wall, Foster-type equivalent network, T-type equivalent network
\end{abstract}

\section{INTRODUCTION}

Stripline 45 degrees bend with input/output stripline of equal width or different width as shown in Fig.2(a) $(b)$ is one of basic components at MIC, whose application examples are shown in Fig.1. So far, the element parameters of equivalent network for stripline bend have been given approximately or empirically, but can not be used for exact analysis and design of stripline circuit. Hence, rigorous equivalent network for stripline 45 degrees bend is strongly needed, which is given by eigenmode expansion method explained in section II. The key step is how to calculate the eigenmode of the planar junction defined in Table 2 . We propose a new idea to calculate that, i.e. 3-stage cascadeconnected stripline configuration and double symmetry with magnetic wall. The detail of our method and calculated results are explained in section III. Once the eigenmodes are calculated by this way, the eigenmodes for any width ratio can be obtained by using the similarity principle of $45 \mathrm{de}$ grees bend.

\section{EQUIVALENT NETWORK BY MODE THEORY}

Stripline 45 degrees bend shown in Fig.2( $a)(b)$ can be modelled to planar circuit with magnetic side wall, which is divided into two planar waveguides and one planar junction. Equivalent network for waveguide is given by multitransmission line and that for planar junction by Foster-type equivalent network derived from eigenmode expansion method. Therefore whole equivalent network is given by Fig.2(c) or vector notation in Fig.2(d), whose network parameters are given in Table 1, after the calculation of eigenmode of 45 degrees bend defined in Table 2. How to calculate the eigenmode is explained in the following section.

\section{CAlCUlation OF THE EIGENMODE FOR PLANAR JUNCTION}

In order to calculate 2D eigenmode for planar junction of stripline 45 degrees bend with equal or different stripline width shown in Fig.2, we propose to use 3-stage stripline

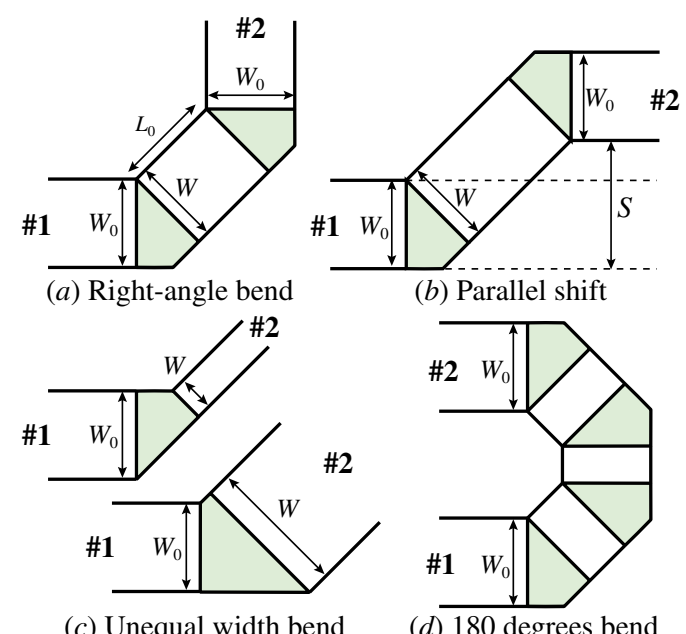

Fig.1 Application of $45^{\circ}$ bend to various stripline circuits

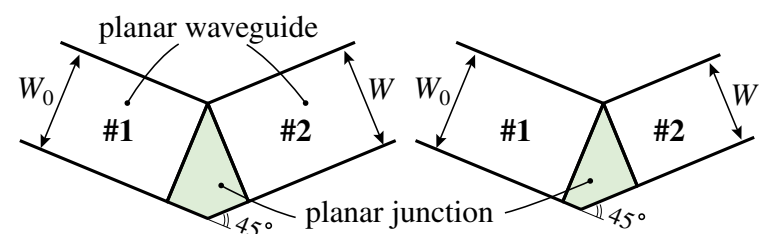

(a) Equal width bend

(b) Different width bend

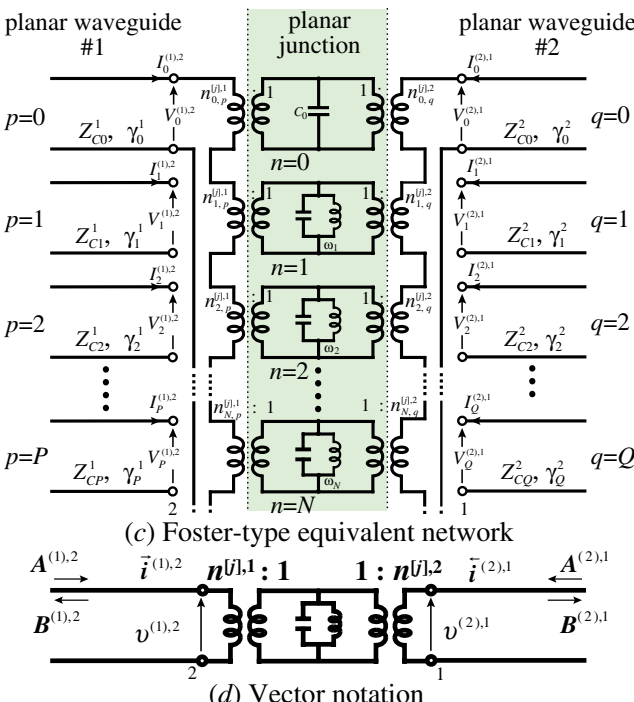

Fig.2 45 degrees bend and its equivalent network

Table 1 Network parameters for equivalent network

$c_{p}^{i}\left(s^{i}\right)=\sqrt{\varepsilon_{p}} \cos \frac{p \pi}{W^{i}} s^{i} \quad(p=0,1,2, \cdots, P)$
$\gamma_{p}^{i}=\sqrt{\left(p \pi / W^{i}\right)^{2}-\omega^{2} \varepsilon \mu}, \quad Z_{C_{p}}^{i}=\frac{j \omega \mu}{\gamma_{p}^{i}} \frac{d}{W^{i}}$
$C_{0}=\varepsilon \frac{S}{d}[\mathrm{~F}] \quad L_{n}=\frac{\varepsilon \mu}{k_{n}^{2}} \frac{1}{C_{0}}[\mathrm{H}] \quad \omega_{n}=\frac{k_{n}}{\sqrt{\varepsilon \mu}}$
$n_{n p}^{i}=\frac{1}{W^{i}} \int_{0}^{W^{i}} \varphi_{n}(x, y) c_{p}^{i}\left(s^{i}\right) d s \quad \boldsymbol{n}^{[j], i}=\left[n_{n_{p}}{ }^{i}\right]$


and double symmetry with magnetic wall. Four planar junctions of stripline 45 degrees bend are connected in back to back as shown in Fig.3(a), then whole structure can be understood as 3 striplines are connected in cascade as shown in Fig.3(b), whose equivalent network is given in Fig.3(c) or $(d)$ in vector notation consisting of three multi-transmission lines and two multi-port ideal transformers. Mode admittance matrix looking toward leftside at port 2 of \#3 waveguide $\overline{\boldsymbol{Y}}_{i n}^{(3), 2}$ can be derived based on definition of port mode voltage/current column matrix and their relation given by network theory. Therefore, eigenvalue equation become

$$
\overline{\boldsymbol{i}}^{(3), 2}=\overline{\boldsymbol{Y}}_{\boldsymbol{i n}}^{(3), 2} \cdot \boldsymbol{v}^{(3), 2}=\mathbf{0} \text {. }
$$

Eigenmodes for 3 -stage configuration can be classified into 4 types of mode distribution because of double symmetry explained in Fig.4. Eigenmodes for $45^{\circ}$ bend are obtained by selecting mode distribution which satisfies magnetic wall symmetry about $A-A^{\prime}, B-B^{\prime}$. For example, calculated eigenvalues up to 20 th and eigenmodes up to 9 th for three-stage configuration with equal stripline width $(R=1.0)$ are shown in Table 3 and Fig.4. We can select three modes $(n=3,6,9)$ from Fig.5, which have double magnetic wall symmetry.

Table 2 Eigenfunction of planar junction

$$
\frac{\partial^{2} \varphi_{n}}{\partial x^{2}}+\frac{\partial^{2} \varphi_{n}}{\partial y^{2}}+k_{n}^{2} \varphi_{n}(x, y)=0
$$

where $\nabla_{t} \varphi_{n} \cdot \boldsymbol{n}=\frac{\partial \varphi_{n}}{\partial n}=0 \quad$ (Magnetic wall)

$$
\begin{gathered}
k_{0}=0 \leq k_{1} \leq k_{2} \leq \cdots \quad \text { (Eigenvalue) } \\
\frac{1}{S} \iint_{S} \varphi_{n}(x, y) \cdot \varphi_{m}(x, y) d x d y=\delta_{n m}
\end{gathered}
$$

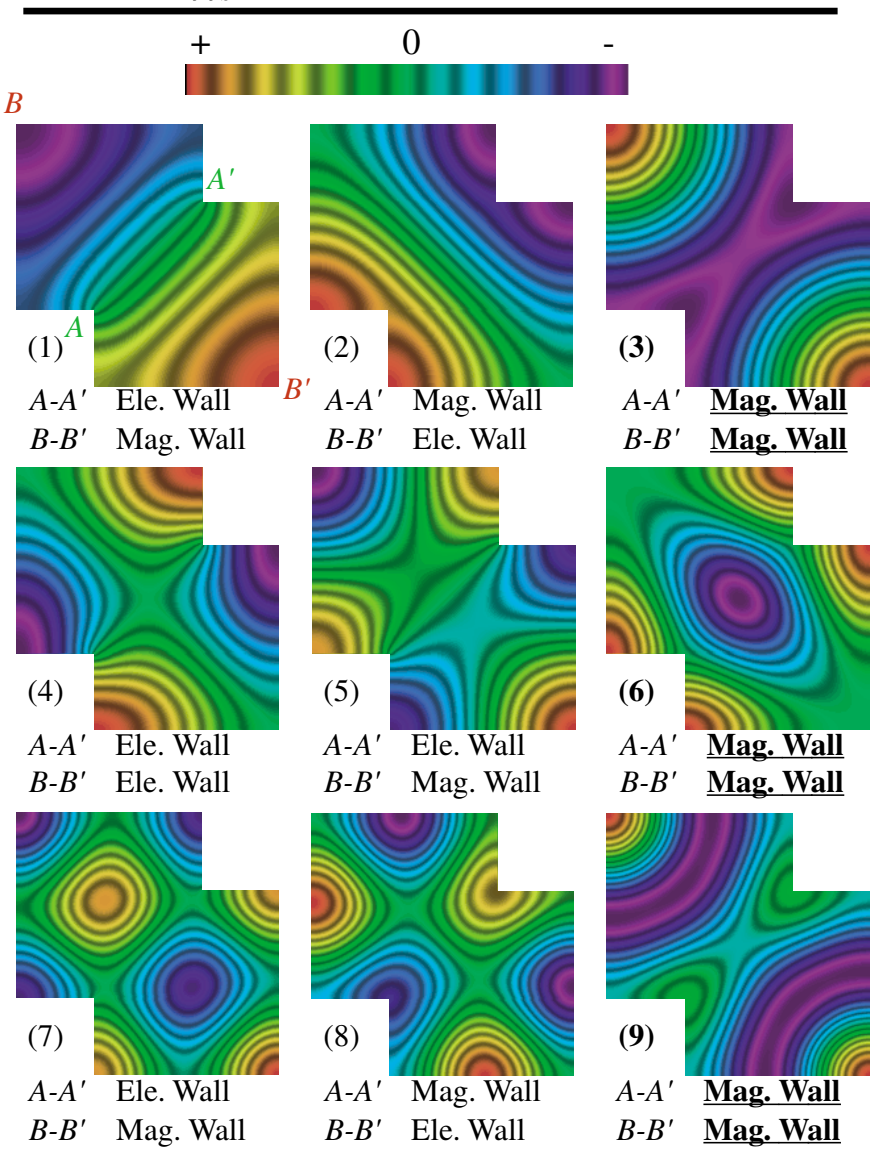

Fig.4 Calculated voltage distribution of eigenmode for 3-stage cascade-connected circuit (a) Double symmetries

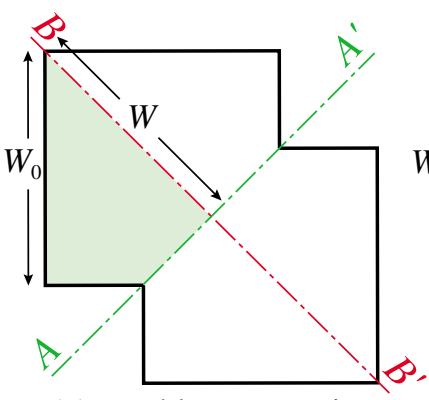

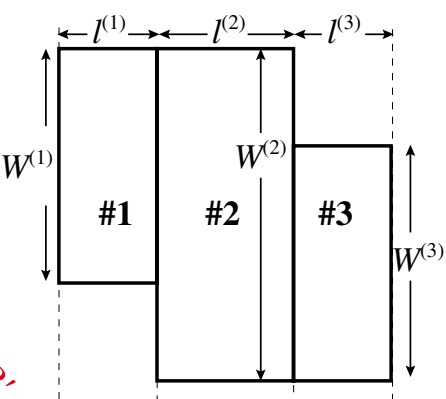

(b) 3 stage connection

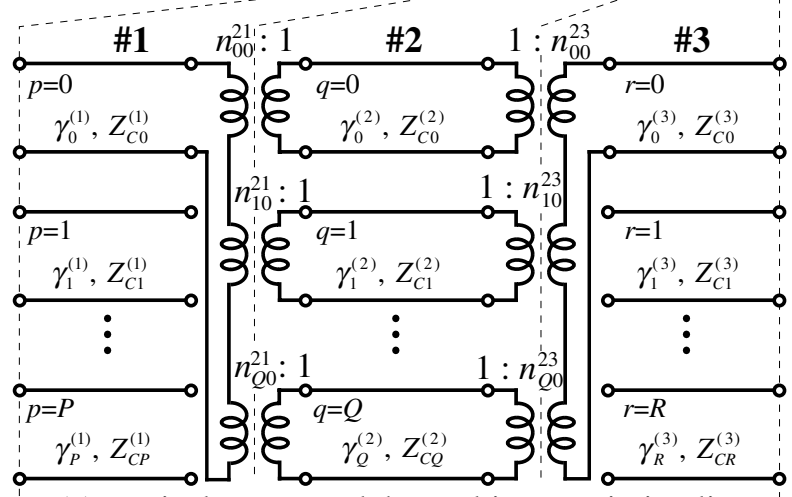

(c) Equivalent network by multi-transmission line and multi-port ideal transformer

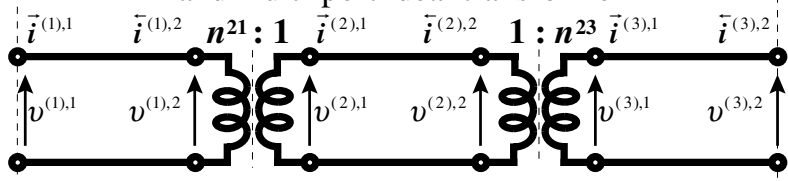

(d) Vector representation

Fig.3 Stripline 3-stage cascade-connected configuration and its equivalent network

Table 3 Eigenvalue of
planar junction
(width ratio $R=1.0)$


By this way, we can select mode distribution and corresponding eigenvalues, whose results are summarized in Table 3 by bold number and in Fig. 5 for $45^{\circ}$ bend (higher modes are added in Fig.5). In this way eigenvalues $k_{n} W$ up to 30 are calculated as a function of width ratio $R=W / W_{0}$ and shown in Fig.6. Eigenvalues in Fig. 6 can be calculated only $1 / \sqrt{2}<R<\sqrt{2}$, but the other ranges $R<1 / \sqrt{2}$ (narrow output line) or $R>\sqrt{2}$ (wide output line) can be derived by using the similarity principle in Fig.7. Therefore, the eigenvalue ranged from $R=0.0$ to $\infty$ can be obtained but only $R=0.0 \sim 3.0$ are shown in Fig.8.

\section{FREQUENCY CHARACTERISTICS}

The calculated eigenmode of stripline $45^{\circ}$ bend gives the network parameter of Foster-type equivalent network by Table 1 . Then, mode impedance is given by following equation and frequency characteristics is shown in Fig.9.

$$
Z_{p, q}^{i, j}=-j \frac{1}{C_{0}} \sum_{n=0}^{N} \frac{\omega}{\omega^{2}-\omega_{n}^{2}} n_{n, p}^{i} n_{n, q}^{j}
$$

It turns out that complete transmission for equal input/out stripline width $(R=1.0)$ and incomplete transmission for unequal width $(R \neq 1.0)$ at low frequency because of reflection due to unequal width. Also, less width ratio $R$, wider bandwidth, because of small planar junction. Phase delay $\varphi_{21}$ and effective length of 45 degrees bend are calculated and

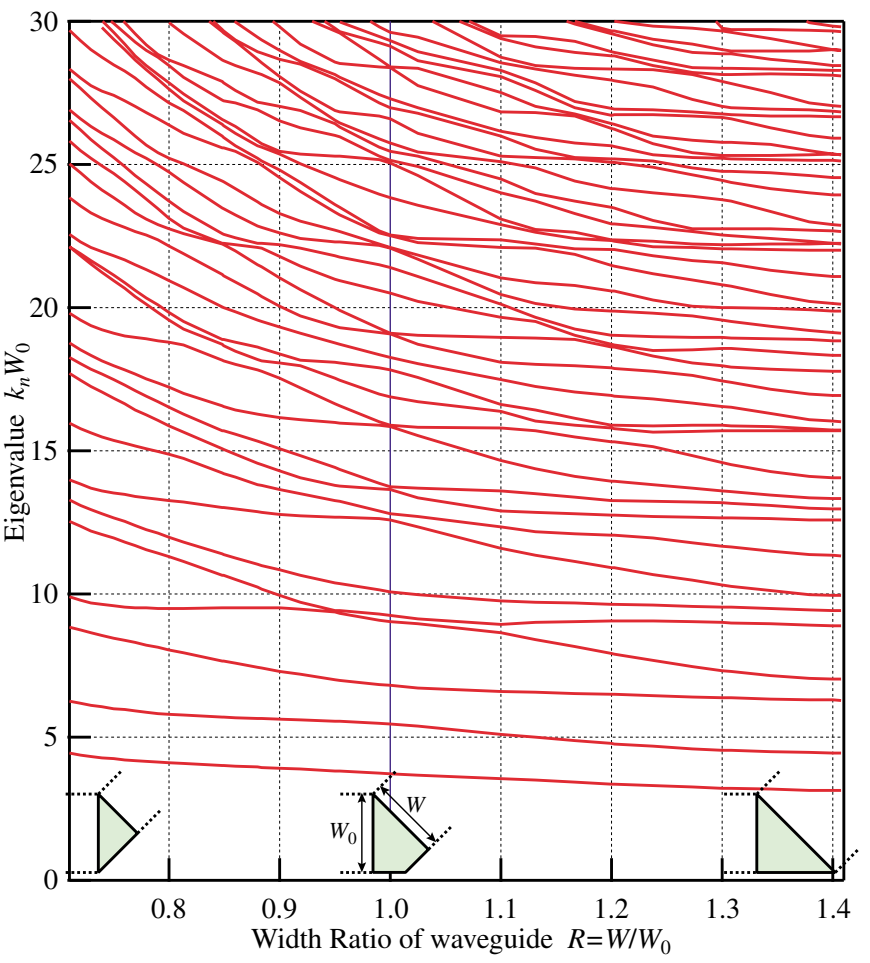

Fig.6 Calculated eigenvalue $k_{n}$ of planar junction (45 degrees bend) $(1 / \sqrt{2}<R<\sqrt{2})$

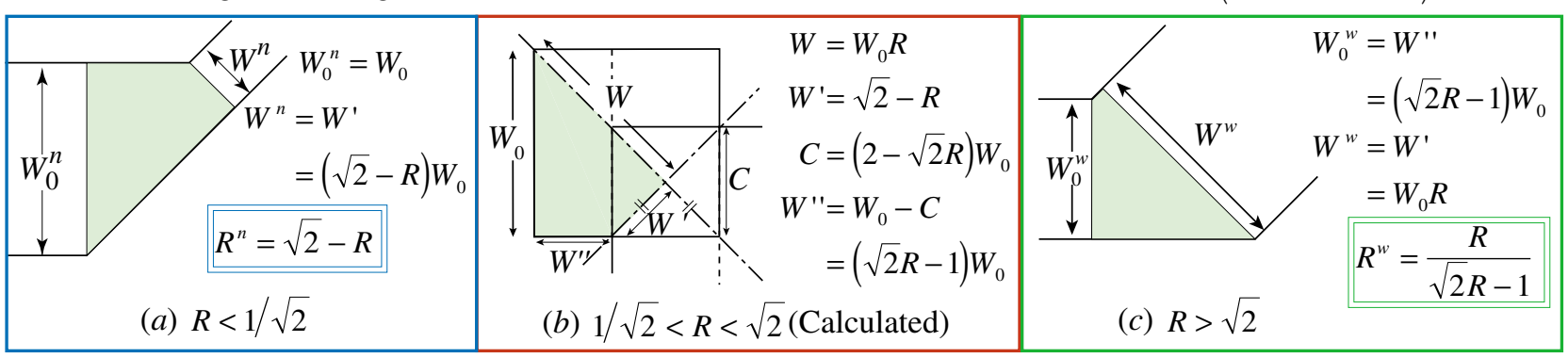

Fig.7 Derivation of the eigenmodes for any width ratio using similarity principle

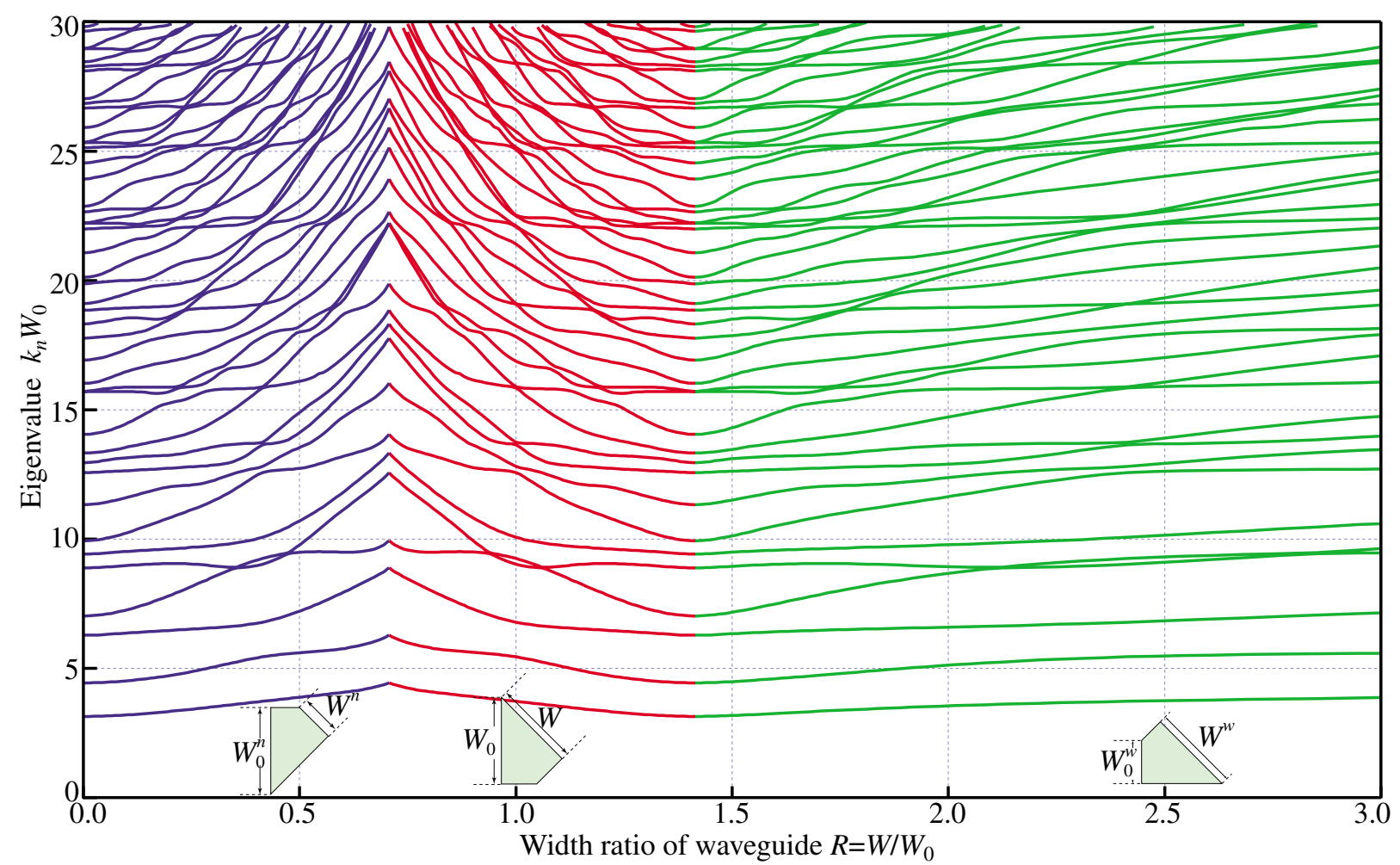

Fig.8 Eigenvalue for various width ratio $R$ 


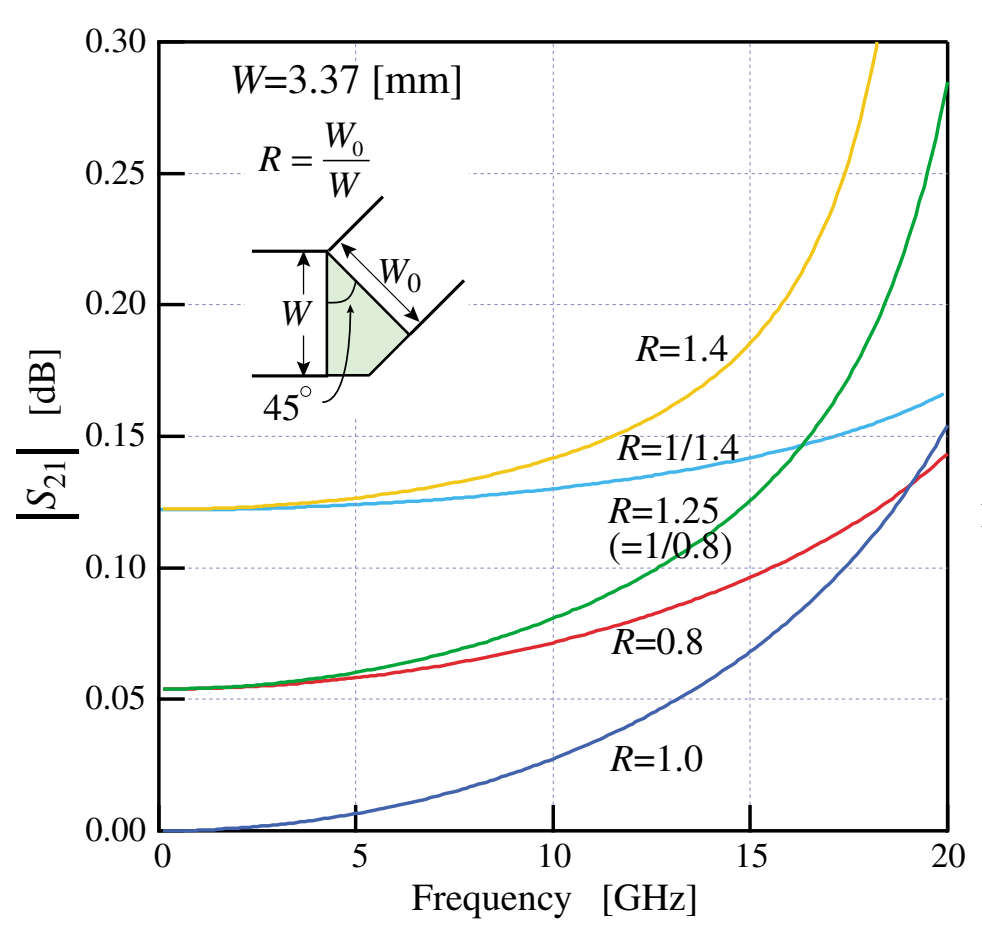

Fig.9 Frequency characteristics for 45 degrees bend with equal width $(R=1.0)$ and different width $\left(R=W_{0} / W\right)$

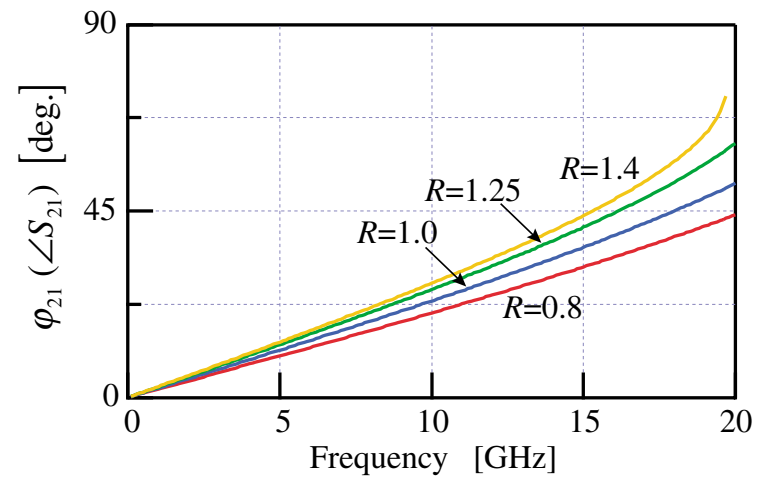

Fig.10(a) Phase delay $\varphi_{21}\left(\angle S_{21}\right)$ of $45^{\circ}$ bend for various $R$

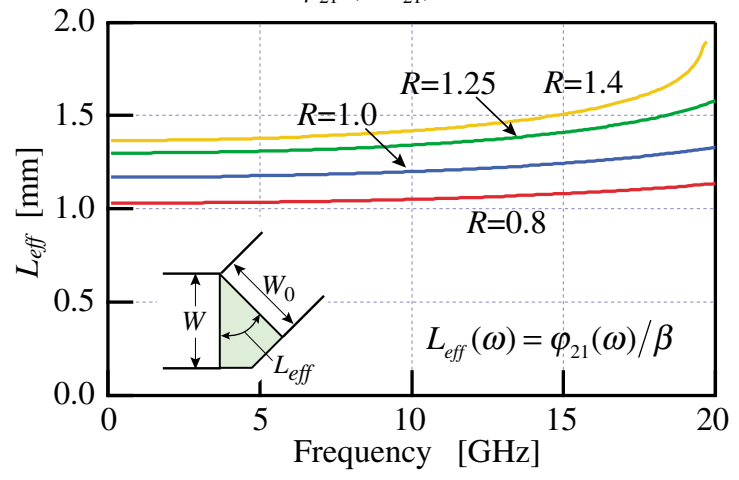

Fig.10(b) Effective length $L_{e f f}$

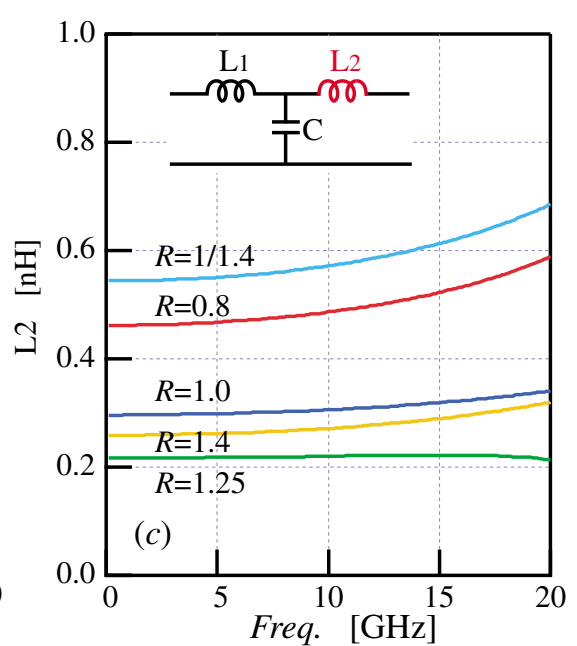

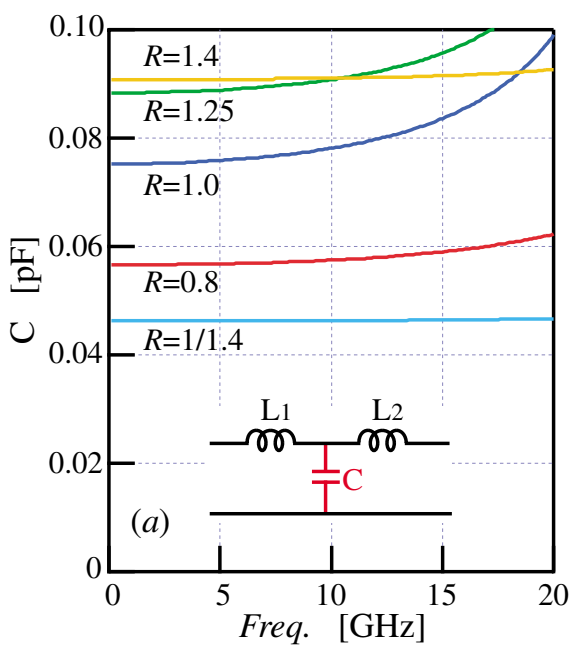

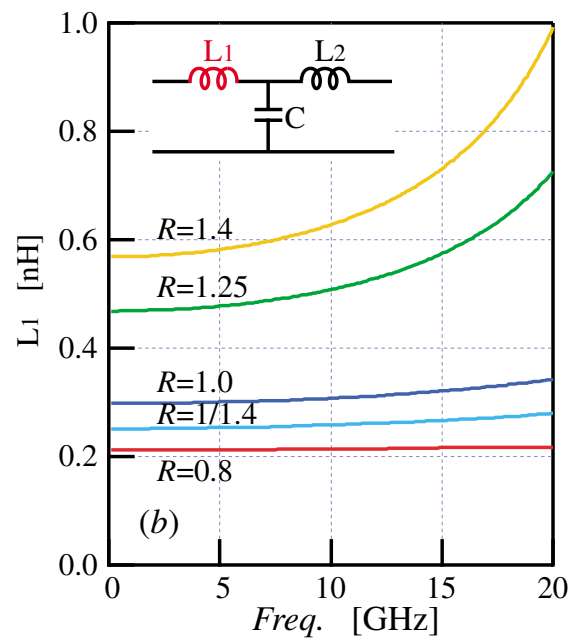

Fig.11 Network parameter of T-type equivalent network (a) Capacitance $(b)(c)$ Inductance

Table 4 Comparison of capacitance

\begin{tabular}{|c|c|c|}
\hline$R$ & $C[\mathrm{pF}]$ & $C^{\prime}[\mathrm{pF}]$ \\
\hline 1.25 & 0.0883956 & 0.0883958 \\
\hline 1.0 & 0.0752587 & 0.0752590 \\
\hline 0.8 & 0.0565732 & 0.0565733 \\
\hline $1 / 1.4$ & 0.0463403 & 0.0463403 \\
\hline
\end{tabular}

shown in Fig.10(a) and $(b)$, respectively.

\section{T-TYPE EQUIVALENT NETWORK}

45 degrees bend can be given by T-type equivalent network shown in Fig.11. Network parameter of T-type equivalent network (C, L1, L2) are calculated and shown in Fig.11, which is derived from mode impedance for Foster-type equivalent network and effective dominant impedance matrix for LC circuit. L1 and L2 become equal at $R=1.0$ and thier difference increase with increasing width ratio. Table
4 compared with $C$ of T-type equivalent network and capacitance $C^{\prime}=\varepsilon_{0} \varepsilon_{S} S / d$ of planar junction of 45 degrees bend around $\mathrm{DC}$, and agreed well.

\section{CONCLUSION}

Novel calculation method of eigenmode for stripline 45 degrees bend is proposed and practically carried out, which can give Foster-type equivalent network of stripline 45 degrees bend. Wideband frequency characteristics of 45 degrees bend are calculated for various width ratio $R$, which can lead to the discussion of the optimum dimension.

\section{REFERENCES}

[1] A.A.Oliner "Equivalent circuits for discontinuities in balanced strip transmission line"

[2] T. Hiraoka, K. Hamatani, J.P. Hsu "Foster-type equivalent network for stripline 45 degrees bend based on calculation of eigenmode and its application" Proceeding of CJMW2004, Pas sive circuits and devices A-1-10 pp33-36 